\section{Absorption Spectra of Excited Atoms and Excited Ions produced by Isothermal Flash Photolysis of Metal Carbonyls in the Gas Phase}

SPIN-ORBIT multiplets of atoms in ground electronic states are particularly interesting for the investigation of electronic energy transfer; simple thoorotical considerations indicate that the relaxation rates correlate with the magnitude of the internal energy changes ${ }^{1}$. Measurements of the formation and docay of excited selenium $\left(4^{3} P\right)$ and iodine $\left(5^{2} P\right)$ have already been reported ${ }^{2,3}$. We have recently shown that the flash photolysis of iron pentacarbonyl $\left(\mathrm{Fe}(\mathrm{CO})_{5}\right)$, which has been investigated in some detail, and of nickel carbonyl $\left(\mathrm{Ni}(\mathrm{CO})_{4}\right)$, can be used to produce excited atoms in gases under isothermal conditions. Spin-orbit relaxation rates can be measured by kinetic spectroscopy, before polymerization of the normal atoms occurs. The method is general to volatile transition-metal compounds, and the observations therefore revoal numer. ous experimental systems for onergy transfer studies.

A flash-photolysis apparatus of standard design produced a 1700-J light pulse in $5 \times 10^{-5}$ sec, and absorption spectra were recorded with a Hilger Medium Quartz Spectrograph. In experiments with $\mathrm{Fe}(\mathrm{CO})_{5}$ at pressures of $0.005-0.001 \mathrm{~mm}$ of mercury, and argon added to a total of $10-50 \mathrm{~mm}$ of mercury, the disappearance of the far ultra-violet continuum of the carbonyl was complete in the first $3 \times 10^{-5}$ sec of the flash. The absence of any significant rise in temperature may be shown by calcula. tion, assuming each $\mathrm{Fe}(\mathrm{CO})_{5}$ molecule to absorb five light quanta at $2000 \AA$, or else demonstrated experimentally by deducing tho equilibrium concentration in the $a^{5} D_{4}$ and $a^{5} D_{3}$ states from the observed line intensities in absorption and the published oscillator strengths ${ }^{4}$.

Table 1 shows the states of atomic iron detected in absorption in flashed $\mathrm{Fe}(\mathrm{CO})_{5}$ /argon mixtures. 'The energies correspond to the lowest of the spin-orbit multiplets ${ }^{5}$.

The highly excited levels rapidly decay to populate the spin-orbit components of the $a^{5} D$ state; relaxation of the $a^{5} D$ multiplets occurs in $10^{-4}$ sec. The calculated tempera. ture rise, the rapid decay of the excited atoms, and the increased rate of decay caused by addition of traces of polyatomic gases prove that the extent of electronic excitation is greatly in excess of a Boltzmann distribution at the translational tomperature, which remains close to $300^{\circ} \mathrm{K}$. (With partial pressures of $\mathrm{Fe}(\mathrm{CO})_{5}$ of the order of $0.1 \mathrm{~mm}$ of mercury, relaxation is extremely fast and the population in the lower electronic states is due to a substantial temperature rise.) Provided the $\mathrm{Fe}(\mathrm{CO})_{5}$ is completely destroy'ed by the flash, the populations in the $a^{5} D$ states are independent of the energy of the flash, and the most highly excited distributions occur at the shortest delay times. Photodissociation evidently produces atomic iron in a range of electronic states, for example:

$$
\mathrm{Fe} \mathrm{CO}+h_{\nu} \rightarrow \mathrm{Fe}^{*}+\mathrm{CO}
$$

Our original objective was to measure the rate of the $a^{5} D_{3} \rightarrow a^{5} D_{4}\left(416 \mathrm{~cm}^{-1}\right)$ transition in argon, and the probability of deactivation has been recorded as $0.2 \times$ $10^{-4}$ at $300^{\circ} \mathrm{K}$. This is significantly slower than vibrational relaxation of non-hydrides, if the same energy is transferred to translation.

Flash photolysis of $\mathrm{Fe}(\mathrm{CO})_{5}$ provides the first example of the production of ions in a gas at concentrations $\left(\sim 10^{12}\right.$ ions/c.e.) suitable for measurement by kinetic absorption spoctroscopy. Fig. I shows the formation and decay of $\mathrm{Fe}^{+}\left(a^{6} D\right)$. The relative populations of the five multiplets

\begin{tabular}{lclc}
\multicolumn{4}{c}{ Table 1 } \\
State & Energy (cm-1) & State & Energy (cm $\left.{ }^{-1}\right)$ \\
$a^{5} D$ & 0 & $a^{3} H$ & 19,360 \\
$a^{5} F$ & 6,928 & $b^{3} H$ & 20,641 \\
$a^{8} F$ & 11,978 & $a^{3} F$ & 21,715 \\
$a^{8} P$ & 17,550 & $Z^{3} F$ & 22,650 \\
$a^{3} P$ & 18,378 & $b^{3} P$ & 22,838 \\
$Z^{7} D$ & 19,350 & $Z^{7} P$ & 23,711
\end{tabular}

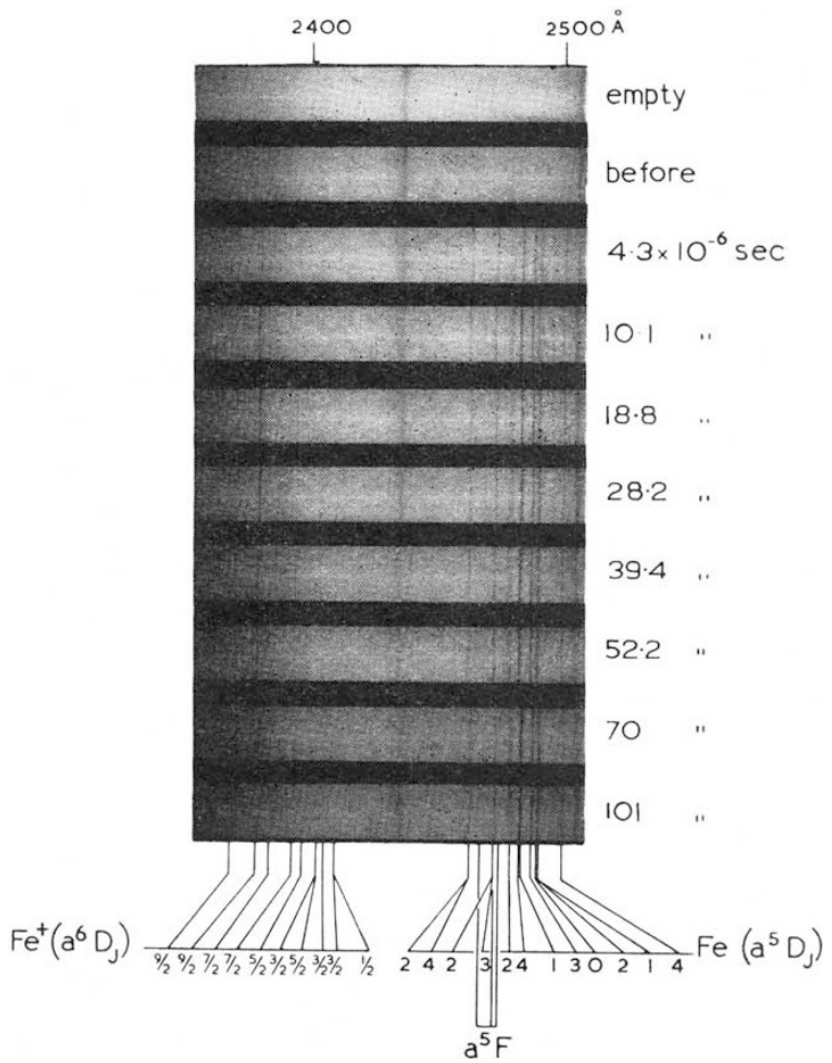

Fig. 1. Formation and decay of $\mathrm{Fe}^{+}\left(a^{6} D\right)$ in flashed $\mathrm{Fe}(\mathrm{CO})_{5}$ vapour

vary during and following the flash. We have also shown that the total concentration of ions increases linearly with flash energy. Ultra-violet radiation at the 1850 A oxygen cut-off is $10,000 \mathrm{~cm}^{-1}$ short of the $7.40 \mathrm{eV}$ ionization potential of atomic iron in the $a^{5} D_{4}$ state. However, all states above $a^{5} F$ suffer ionization by secondary light absorption. The decay rate of the ions increases with increasing pressure, which may suggest that removal is by three body processes.

We have made preliminary moasurements of the non. Boltzmann electronic-distribution of atomic nickel, produced by flash photolysis of $\mathrm{Ni}(\mathrm{CO})_{4}$.

A. B. Callear

R. J. OLDMAN

Physical Chemistry Laboratory, University of Cambridge.

1 Callear, A. B., App. Optics, Second Laser Suppl., 145 (1965).

Callear, A. B., and Tyerman, W. J. R., Trans. Faraday Soc., 61, 2395 (1965). ${ }^{3}$ Donovan, R. J., and Husain, D., Nature, 206, 171 (1965).

4 Corliss, C. H., and Bozman, W. R., Transition Probabilities, Nar. Bur. Stand. Monograph $53(1962)$.

s Moore, C. E., Atomic Energy Levels, Nat. Bur. Stand. Circular 467, 2 (1952).

\section{Dynamic Fatigue in Glasses}

THE static fatigue of glasses is a woll-known and well-documented phenomenon (see, for example, ref. 1), but the only investigation of eyclic loading fatigue known to tho authors is that of Gurney and Pearson ${ }^{2}$, who reported a negative result. Perhaps in view of this rosult and of the near ideal brittle behaviour of glasses the investigation of cyclic loading fatigue appears to have been neglected. Recent results obtained in these laboratories as part of an extensive investigation of the strength of fused silica have indicated that a real cyclic fatigue effect may exist in this material. Circumstances have prevented a full investigation and confirmation of the effect, but in view of the importance of the problem the preliminary results would seem worth reporting here.

In the upper part of Fig. 1 a comparison is made between the static fatigue life under constant tensile 